\title{
L'esthétique Théâtrale De L'objet Récupéré : De La Matérialité Quotidienne À La Représentation Scénique
}

\section{Tadili Khalid}

Doctorant au laboratoire, Théâtre et Arts de la Scène, Université Ibn Toufail, Faculté des Lettres et des Sciences Humaines, Kénitra, Maroc

Doi:10.19044/esj.2018.v14n26p265 URL:http://dx.doi.org/10.19044/esj.2018.v14n26p265

\begin{abstract}
This paper focuses on highlighting the particular contribution of the recovered object in the change of artistic techniques and the aesthetic modalities of theatrical representation. It is an opportunity to emphasize this alliance between everyday materiality and the space of the scenic representation in the form of the recovery of the forgotten and an attribution of a second life which is relative to objects marked by obsolescence. Objects loaded from reality, familiar use, and social sharing acquired rhetorical functioning captivated by their use in unusual space. Our approach consists of stopping on this particularity of the recovered object from a comparative study of its different uses by the directors and the playwrights. This is done in order to highlight the artistic potentialities which it generates and the various aesthetic reports that it triggers in the theatrical field. This type of representation highlights the fight against the obsolescence in favor of a new spectacular load so as to mobilize the spectators' imagination. For this purpose, it seems to us that the recovered object is able to find the base of its aesthetic representation. It is about a reflection object which makes the usual the origin of a rhetorical expressivity in order to place it at the very center of the theatrical creation.
\end{abstract}

Keywords: Daily materiality, ludic potential, the aesthetics of recovery, metamorphose, rhetoric

\section{Résumé}

Dans cet article, nous mettons l'accent sur l'apport particulier de l'objet récupéré dans le changement des modalités esthétiques de la représentation théâtrale. C'est une opportunité pour mettre en relief cette alliance entre la matérialité quotidienne et l'espace de la représentation scénique sous forme 
de relations qui privilégient la récupération de l'oubli et l'attribution d'une seconde vie, cette fois-ci ludique, aux objets marqués par la désuétude. Des objets chargés du réel, d'usage familier et de partage social se dotent de fonctionnements rhétoriques captivants par leurs déplacements à un espace inhabituel. Notre démarche consiste à s'arrêter sur cette particularité de l'objet récupéré à partir d'une étude comparative de ses différents usages par les metteurs en scène et les dramaturges afin de mettre en valeur les potentialités artistiques qu'il génère et les divers rapports esthétiques qu'il déclenche dans le champ théâtral. Cette sorte de représentation favorise la lutte contre l'obsolescence des objets en faveur d'une nouvelle charge spectaculaire mobilisant également l'imaginaire du spectateur. C'est à ce prix, nous semblet-il, que l'objet récupéré a pu fonder les assises de sa représentation esthétique. Il s'agit, en effet, d'un objet de réflexion qui rend le quotidien l'origine d'une expressivité rhétorique et le place au centre même de la création théâtrale.

Mots-clés: La matérialité quotidienne, potentiel ludique, l'esthétique de la récupération, métamorphose, rhétorique

\section{Introduction}

L'objet théâtral reflète une source d'innovation qui n'a pas cessé d'influencer les dramaturges et les metteurs en scène depuis l'antiquité jusqu'à notre ère contemporaine. A cet égard, la simplification des volumes chez Edwardt Gordon Craig, la recherche d'une circulation et les hauteurs chez Adolphe Appia, le dépouillement scénique chez Jacques Copeau, la biomécanique de Vsevolod Meyerhold, la distanciation chez Bertolt Brecht, la pauvreté de la part de Jerzy Grotowski au bio-objet de Tadeusz Kantor, forment, en somme, une pluralité de possibilités d'apparition de l'objet sur scène. Cette diversité révèle que la présence de l'objet dans n'importe quelle thématique échappe à toute gratuité et suppose une attention profonde de l'usage que l'on en fait.

En effet, la particularité de l'objet théâtral réside dans le fait qu'il entretient un réseau de relations avec deux univers différents, l'un fictif et l'autre réel. L'objet est considéré en tant que tel dans la réalité et ce qu'il prend en charge dans la fiction. Il est un « être là » de par sa présence tangible et, en même temps, une figure symbolique. Une telle dichotomie nécessite, en quelque sorte, l'oubli de sa charge connotative- valorisante ou dévalorisante- dans la vie quotidienne au profit d'une orientation poétique dans laquelle son pouvoir d'évocation et de suggestion prime sur toute autre considération habituelle d'où la particularité de ses approches esthétiques.

À ce titre, une manipulation de l'objet qui nous semble retenir un intérêt primordial surtout dans les représentations modernes et contemporaines est celle relative à la conception de l'objet retrouvé, ou bien ce qui est considéré 
sous les acceptions de «l'objet vrai », «l'objet authentique » et « l'objet pauvre ». Il s'agit, en effet, d'une considération esthétique qui se trouve fortement ancrée dans le cadre de la vie quotidienne et qui tente de créer un nouveau rapport avec les objets, d'ordinaire, utilisés dans des perspectives uniquement fonctionnelles. Quant à leur référence, elle est commune dans l'esprit de chaque utilisateur. Autrement-dit, ces objets qui ont déjà leur propre vécu ou qui ont déjà « une expérience sociale » se voient en mesure d'innover la pratique théâtrale par des procédés inhabituels et d'esthétiser, en même temps, notre rapport avec notre univers quotidien.

Par le biais de cette perspective, il serait opportun de démêler cette nouvelle alliance entre la matérialité quotidienne et l'espace de la représentation scénique. Une corrélation particulière nous emporte dans une dimension purement poétique qui ne peut être que le résultat d'un imaginaire subtil de la part des dramaturges et des metteurs en scène. Un tel imaginaire redonne une seconde vie à des objets manufacturés en référence à la société de consommation mais, cette fois-ci, dans une sphère ludique qui les charge de potentialités évocatrices.

En ce sens, il convient de rappeler l'expérience de Bertolt Brecht( 1972) qui prône pour la mise en scène d'objets " usés », voir même "des objets arrachés à un chantier de démolition »; en quelque sorte, des objets qui ont déjà servi à une fonctionnalité quelconque et qui sauvegardent la marque de leur usage. De même, Meyerhold opte pour le recours à des objets « authentiques » dont la valeur expressive détermine leur manipulation sur la scène. Dans la même perspective, Tadeusz Kantor se contente « d'objets réels », pour créer une vision artistique à partir de leur récupération de l'oubli et de l'engloutissement. D'une visée globale et comme Christian Carignon a suggéré, ce sont «des objets que l'on ne voit plus à force de les voir » (Justine Duval, 2012). Bref, ces objets chargés du réel, d'usage familier et de partage culturel se dotent de fonctionnements rhétoriques captivants par leurs déplacements scéniques.

De là, il nous paraît essentiel de reconnaître, à partir de cette pluralité d'usage des objets relatifs à la pratique quotidienne dans le champ de la représentation scénique, leur apport dans la diversité de la pratique théâtrale. En quelque sorte, comment ce genre d'objets participe-t-il à innover de nouvelles pratiques de jeu ? Encore, comment l'objet récupéré se différenciet-il de n'importe quel autre objet théâtral ? D'une autre façon, qu'est ce qu'un objet récupéré sur scène ? En plus, par l'entremise de cette mise en jeu de l'objet récupéré sur l'espace théâtral, ne s'agit-t-il pas d'une remise en question à propos de la relation de l'humain avec son environnement immédiat? Et, ce constat de déplacement sur scène, ne modifie-t-il pas notre regard sur ce genre d'objets dans la vie quotidienne ? 
Pour effectuer l'analyse de ce rapport particulier qui englobe la matérialité quotidienne et la représentation scénique, nous allons nous pencher sur les axes suivants :

- Les différentes acceptions ludiques de l'objet récupéré.

- L'objet récupéré et l'attachement à l'humain.

- L'esthétique de la représentation de l'objet récupéré.

\section{Critères d'identification de l'objet récupéré}

En effet, la particularité de l'objet dit récupéré réside dans le fait que le spectateur maîtrise son utilité et son fonctionnement ordinaire dans la vie quotidienne. Mais sa manipulation, cette fois-ci, est d'ordre fictionnel. Ce constat met le spectateur dans une situation de doute entre ce qu'il reconnaît préalablement et ce qu'il voit sur la scène. Aussi cet écart produit-il à la fois une action étrange et une sensation agréable par le fait de rompre l'horizon d'attente du spectateur. En quelque sorte, ce qu'est visé par cette opération est cet effet surprenant qui constitue l'essentiel de la pratique théâtrale.

$\mathrm{Ce}$ processus ludique permet de transformer une réalité concrète et quotidienne en une autre simultanément imaginaire, signifiante et esthétique. Une telle démarche rend l'interprétation de la part des spectateurs une opération délicate et, en même temps, catalyseur d'un regard particulier sur des éléments donnés à voir dans une configuration matérielle inhabituelle. Dans cette optique, il est question de déchiffrer ce lien entre l'ordinaire ancré dans le réel et l'imaginaire cristallisé par une visionde métamorphose de celuici. Cette mise en relation entre les choses reflète, d'une part, le faire-perceptif du spectateur et, d'autre part, le faire- réflexif des metteurs en scène qui tentent de charger le familier, le trivial et le banal par des connotations représentatives. D'une manière générale, il s'agit d'une invitation à reconfigurer les choses selon une conception théâtrale qui consiste à passer du visible à l'invisible.

Il en ressort donc qu'il s'agit d'un processus de création basé sur le déplacement de la matérialité quotidienne, ce qui mobilise l'imaginaire du spectateur et redonne une seconde vie à des produits manufacturés. Une telle représentation favorise, d'une part, la lutte contre l'obsolescence et la mise au rebut des marchandises et, d'autre part, nous rappelle l'affiliation au Readymade dans lequel l'objet est utilisé sous sa forme originelle, c'est-à-dire, sans modification ou préparation pour la représentation. Cela révèle qu'il n'est pas question, ici, de représenter seulement l'objet mais de le présenter en vue d'une signification à rechercher selon la conception du réalisateur de cet acte. De là, l'objet selon Anne Ubersfeld (1984) peut « devenir ce que veulent à un moment déterminé du spectacle, à la fois l'acteur et l'action dramatique ».

Plusieurs considérations innovantes dans la pratique théâtrale nous amènent à nous demander ce qui seraient véritablement les critères d'un objet 
récupéré de l'usage quotidien à la manipulation théâtrale. En ce sens, il convient de préciser que c'est Bertolt Brecht qui a utilisé premièrement ce genre d'objet sur scène. Selon sa propre conception, ces objets se démarquent clairement par une mémoire d'usage familier. Il est question d'objets sauvés de l'oubli et de la disparition parce qu'ils étaient dépassés technologiquement, voire même, hors d'usage. Dans ce cas, les objets deviennent des témoins des actions humaines ordinaires. Mais l'indifférence manifestée à leurs égards, empêche de les considérer en tant que des éléments porteurs d'une charge esthétique, d'où le fait que la particularité de l'activité théâtrale consiste à procéder en une pratique d'appropriation visant à rendre l'insolite un élément catalyseur de visions artistiques.

Une remarque importante à souligner dans cette perspective est que la visée de Bertolt Brecht consiste à utiliser certainement un objet « vrai et authentique » mais pour produire une singularité à celui-ci sur la scène. D'une certaine façon, il s'agit d'un objet ordinaire mais qui déclenche, en même temps, tout un processus imaginaire et réflexif pour déterminer véritablement sa nouvelle fonction spectaculaire. À ce niveau, Bertolt Brecht (1978) est loin de l'idée de manifester «une redondance illustrative » de la réalité, étant donné qu'il privilégie une représentation qui se base sur le principe de la distanciation pour « une reproduction qui certes fait reconnaître l'objet, mais qui le fait en même temps paraître étranger ».

Suite à ce chevauchement, l'objet offre au spectateur le plaisir d'une vérité insaisissable, à la fois réelle mais qui nécessite un accomplissement par l'imaginaire, d'où l'orientation d'éveiller la conscience de celui-ci « de la torpeur d'une illusion identificatrice »,(Jean-Luc Mattéoli, 2001). L'objectif principal de la représentation théâtrale est donc d'inviter le spectateur à la réflexion, comme le suggère Bertolt Brecht (1978); "aux époques où une partie des hommes introduit la confusion entre les choses, on a souvent avantage à mettre ostensiblement la pensée en vedette par des signes. Le théâtre doit alors indiquer combien il est nécessaire de penser ». À partir de cela, on peut déduire que de la confusion nait la polysémie de l'objet récupéré sur la scène.

Dans cette même perspective, Tadeusz Kantor (1990) annonce qu'il s'agit, «d'un objet misérable, pauvre, incapable de servir dans la vie, bon à jeter aux ordures, débarrassé de sa fonction vitale, appelant la pitié et l'émotion. ». Ces critères révèlent qu'un tel objet n'a plus d'utilité dans la vie quotidienne et que son itinéraire d'usage est arrivé à un stade terminal marqué par l'oubli et le désintéressement. De quelque manière, cet objet a perdu toutes les possibilités d'un fonctionnement commun et se trouve dans les poubelles. C'est pourquoi la recherche dans « un chantier de démolition » serait le lieu propice pour retrouver le matériel nécessaire à la représentation. En effet, cette situation affreuse dans laquelle se trouve l'objet suscitela pitié de la part des 
êtres humains parce qu'il a partagé avec eux l'espace de leur existence, ce qui signifie que la composante temporelle de l'objet peut être renouvelée par ce geste de la récupération d'une disparition définitive.

Dans cette optique, le projet de la représentation théâtrale favorise une part émotionnelle afin de toucher la sensibilité du spectateur par des images relatives à son tempérament cruel envers les partenaires de sa vie quotidienne. Cette volonté de rendre le moment de la représentation un instant d'analyse psychologique de nos conduites humaines, déclenche parfois les pleurs comme une réaction esthétique. Tout cela s'inscrit dans un projet global, celui d'inventerun théâtre dit de « la mort » parce qu'il redonne égalementla chance à l'objet de retrouver une seconde vie sur la scène.

Autre critère à souligner est celui de Christian Carrignon qui précise qu'il s'agit des « objets reconnaissables par tous », facilement identifiables par le spectateur. De tels éléments sont percevables avant que leur exploitation ne se déploie dans une structure spectaculaire parce qu'ils font partie intégrante du cadre de la vie ordinaire. En effet, ces objets qui sont le reflet de la modernité et de la société de consommation mettent en valeur également la mentalité régnante de l'époque. C'est le temps de l'influence de la technologie industrielle, de la consommation excessive des produits manufacturés et de la remise à rebut des marchandises. Il est question donc des objets qui constituent la sphère d'apparence quotidienne de l'être humain à tel point que peu importe quelle est la démarche analytique de celui-ci, elle ne peut pas se faire sans les éléments illustrant son extériorité. Cela s'explique de la part de Christian Carrignon (2011) par le fait que «Nous sommes les enfants de la société de l'objet, nous pensons à travers l'objet. Il est simple, lisse, beau, pas cher et il nous fascine sous son apparente innocuité ».

Dans cette perspective, les objets ordinaires qui envahissent notre monde sont devenus des catalyseurs de la fiction à force de leur proximité à cette structure du quotidien. Christian Carrignon (2011) ajoute également : «on $a$ fait avec ce qu'on avait; et on avait rien d'hérö̈que », d'où la prolifération des objets banals sur la scène théâtrale ou des objets en plastique industriel qu'il a nommé « en plastoc ». L'essentiel est quela manipulation théâtrale de l'objet devienne une nécessité parce qu'elle répond aux normes de l'époque actuelle. Il s'agit de l'adoption d'une démarche esthétique qui rend la représentation théâtrale de l'objet purement constitutive de notre identité collective, raison pour laquelle on parle du théâtre d'objet comme illustration d'un théâtre de la vie quotidienne.

Il en résulte donc de ces différentes acceptions des objets récupérés, un trait distinctif, celui de l'omniprésence dans tous les cas d'une «biographie sociale » illustrant, d'une part, les différents usages des objets et favorisant, d'autre part, un partage culturel entre le spectateur et le metteur en scène. En effet, la manipulation par l'esprit de ce genre d'objets repose sur la mémoire 
individuelle et collective qui assureune fonction médiatrice entre la charge acquise par une familiarité quotidienne et la recharge spectaculaire par des procédés mettant en valeur l'image poétique.

Une telle démarche permet donc d'identifier l'objet récupéré en tant qu'objet du réel parce qu'il en garde toujours sa charge connotative. Quoiqu'il soit manufacturé en plusieurs exemplaires et symbole par excellence de la société de consommation, l'objet récupéré est vidé de sa charge habituelle par la particularité de l'activité théâtrale, pour le remplir d'une charge spectaculaire. D'une visée globale, la fonction d'usage ordinaire s'éclipse au profit de la manipulation théâtrale.

Ce constat révèle que l'objet peut toujours se métamorphoser en autres choses selon la conception admise par le metteur en scène, ce qui a amené Bertolt Brecht à prôner que l'usure favorise la liberté des potentialités artistiques de l'objet. À ce titre, l'objet retrouvé dans un chantier de démolition a déjà mis fin à son parcours d'usage ordinaire et il est apte à d'autres manipulations sans se soucier du cadre où il serait exposé. L'essentiel est que l'objet échappe à toute considération étroitement technologique pour figurer sur un champ scénique nécessitant, cette fois-ci, un dialogue particulier avec les autres éléments du spectacle.

Ce dialogue exige de la part du spectateur de décoder les nouvelles significations de l'objet indépendamment de toute projection relative à ses référents quotidiens. De ce fait, la lecture de l'objet est intrinsèquement liée à sa prise en considération dans une syntaxe poétique dans laquelle le projet spectaculaire l'emporte sur toute considération fragmentaire. Cela signifie que son appréhension avec les autres éléments de la représentation doit se faire en parallèle avec son articulation à la fois « synchronique et diachronique ». Par cette opération, l'objet donne lieu à la production d'un langage sensible basé sur les signes, les icônes, les symboles..., bref, sur une " resémantisation » rhétorique de la sphère quotidienne.

Il paraît clair que l'objet récupéré est une notion capitale dans la représentation théâtrale par la diversité qu'elle a instauré au niveau des paramètres ludiques sur la scène. Il est question d'un jeu qui mobilise la conscience du spectateur et l'invite à la réflexion, ce qui induit des manières différentes de la manipulation de l'objet. Cette mutation remarquable demeure au service d'un jeu rhétorique dans lequel la présence concrète est aisément identifiable par le spectateur. En revanche, la configuration scénique est fortement liée à un processus de la représentation à la fois mental et imaginaire. Donc, l'acquisition de propriétés esthétiques de l'objet récupéré serait conditionnée par ce geste particulier de l'extraction de l'oubli et de la recontextualisation dans une sphère représentative dans laquelle il retrouve une nouvelle fonction, cette fois-ci, symbolique. 
Cette propriété théâtrale ne peut se réaliser que relativement au nouveau regard attribué aux objets quotidiens. Il s'agit, autrement-dit, d'une volonté implicite d'esthétiser notre rapport quotidien avec eux. Ce constat nous permet de synthétiser que toutes ces acceptions riches de l'objet récupéré, porteur de mémoire, de gestes authentiques et de présence réelle, sont une tentative de retrouver ou «d'éveiller» le sens de l'humain tant oublié par cette forte puissance de la société de consommation et de son rythme effréné de changement et d'évolution.

\section{L'objet récupéré et l'attachement à l'humain}

Cette notion de l'objet récupéré nous révèle que le trait humain est un principe fondateur de cette nouvelle mise en relation spectaculaire des objets. On peut dire que l'objet récupéré nous permet de voir les différentes images illustratives des attitudes humaines. Au début, l'être humain manifeste de l'intérêt crucial à acquérir un objet en vue de l'utiliser dans des fonctions essentielles relatives à ses préoccupations quotidiennes. Mais avec l'influence de la modernité, il l'oublie facilement pour un autre qui répond à d'autres exigences esthétiques. Après une certaine période de désintéressement, il ressent de la nostalgie envers ses objets délaissés. De ce fait, ce trait fluctuant qui caractérise le désir de l'être humain renvoie à une volonté d'instaurer une relation de continuité avec l'objet, malgré quelques moments de rupture. Cela justifie, en grande partie, la tendance à présenter l'objet récupéré sur la scène théâtrale parce que l'être humain récupère également à travers lui ses propres images familières.

Pour illustrer cette situation, il serait utile de s'arrêter sur l'analyse pertinente de Jean-Luc Mattéoli de ce genre d'objets distingués de sa part par la pauvreté. Certes, une telle pauvreté s'explique par leur appartenance à une réalité dégradée et révolue, mais qui met en valeur la richesse de leurs potentialités artistiques et théâtrales. Ces objets envers lesquels on manifeste de l'indifférence, du dégoût et de la désaffection semblent toucher l'émotion du spectateur par une manipulation inhabituelle qui nous rappelle une telle relation humaine avec-eux.

L'objet pauvre sauvegarde toujours une présence humaine à l'image d'un « réceptacle muet» et « un réservoir à mémoire». Mais cette fois-ci la pratique théâtrale rend à l'objet pauvre son langage matériel oublié par l'airain de l'usage fonctionnel. Il s'agit donc d'objet pauvre parce que l'être humain n'a pas encore cherché à l'utiliser et à l'exploiter dans d'autres perspectives en dehors de sa structure de pratique référentielle (Jean-luc Mattéoli, 2007)

«parce que, quotidien, il est dépourvu des traits qui pourraient le hisser à hauteur d'art (pour le sens commun, l'art exclut la trivialité du quotidien), mais il l'est aussi parce qu'on le plaint d'en être réduit à cette extrémitéque constituent la rue, la poubelle, l'ordure ». 
Ce trait de pauvreté est une réaction contre le jugement de beauté étroitement lié au sublime et au raffiné.

De là, on peut préciser que l'objet pauvre est celui qui produit ce mélange entre l'émotionnel et l'indifférence, entre le quotidien et l'esthétique, celui qui critique notre acte du modernisme et nous invite à une réflexion à propos des raisons qui nous ont conduites à ce point de rupture avec des éléments ancrés dans notre histoire quotidienne. Somme toute, il s'agit d'un objet mémoriel et vivant à force de sa mise en récit de l'histoire humaine et de son intégration à nos pratiques vitales et sociales, mais qu'on cherche désormais à anéantir son existence par l'oubli et la manipulation visant à le priver de ce qui constitue sa quintessence (Jean-luc Mattéoli, 2011);

"l'animer, ce serait le priver de cette vie et de cette parole propre qu'il recèle; le métamorphoser, ce serait le rendre invisible, en brouiller "la présence ", voire l'escamoter, et avec elle tout ce que l'objet, en quelque sorte, contient.»

Donc, l'objet pauvre est un élément «étrangéisé » par son caractère dépassé. Par contre, il nous fascine par ses déplacements significatifs d'une structure d'accueil à une autre. C'est la concrétisation d'une relation triadique entre le réel, le vivant et l'humain.

Il en ressort donc que l'objet pauvre est un élément qui résiste à l'engloutissement et à l'obsolescence, et son usage prolifique dans les scènes contemporaines est une revalorisation de ses qualités matérielles. Une telle banalité conservatrice de l'histoire se trouve en mesure de modifier les canons habituels de la représentation théâtrale. Ainsi, on parle de projets d'assemblage et de juxtaposition, aussi, de l'objet partenaire, de l'objet actant et de l'objet lieu selon les conceptions recherchées par les metteurs en scène. Bref, on attribue à des techniques aléatoires une profondeur esthétique.

À ce titre, le théâtre d'objet et de marionnette est l'illustration parfaite de ce renouvellement technique qui redonne à l'activité théâtrale une dimension affective et poétique. C'est alors qu'on peut justifier cette tendance à utiliser des éléments quotidiens dans la représentation scénique par la nostalgie humaine et par la crainte d'une disparition furtive (Jean-luc Mattéoli, 2011);

"Le goût du quotidien au théâtre (mais aussi dans la littérature, au cinéma) est peut être l'envers de l'amnésie à laquelle le public se sent condamné par le règne d'une obsolescence dont il perçoit de plus en plus clairement qu'elle s'applique aussi aux hommes ».

À vrai dire, l'objet pauvre pour Jean-Luc Mattéoli révèle le statut pauvre également de l'être humain. Celui-ci constate que ses créations matérielles avec lesquelles il a partagé tant de moments d'usage fonctionnels et de projections psychologiques risquent une disparition, en quelque sorte, un effacement de son champ de pratique, et qu'il ne peut rien faire pour eux. Cet attachement est certainement le motif essentiel de penser à détourner les 
objets, cette fois-ci, de l'oubli vers une actualisation de leur mémoire et de leur fonction de nature spectaculaire. De cette façon, l'être humain sauve l'objet de l'obsolescence et aussi une partie essentielle de son existence afin de prouver à lui-même qu'il ne risque pas un tel sort inéluctable. Donc, charger l'objet de nouvelles fonctions c'est lui donner de la vie et à l'être humain de l'espoir à changer la réalité des choses même si elle appartient au quotidien.

Cet attachement profond à la manipulation des objets anciens ou leur récupération de la disparition est un principe d'identité de l'être humain fondé sur une expérience directe avec les éléments matériels. En ce sens,n'importe quel contact avec eux laisse une trace ineffaçable dans la mémoire humaine. En outre, le renouvellement des images du passé leur donne une dimension émotionnelle. C'est pourquoi on parle de mémoire sensible lorsqu'on essaie d'actualiser les moments de partage collectif entre l'humain et le matériel. Cependant, la particularité de la représentation théâtrale consiste à opérer ce transfert qu'on peut qualifier de nostalgique par le biais même de l'imaginaire. Il s'agit d'une forme de représentation qui se construit à partir des postulats rhétoriques.

En fin de tout, l'objet récupéré est porteur d'une valeur nostalgique qui concentre en lui l'usage quotidien, l'imaginaire et la manipulation transformatrice. En réalité, c'est une concrétisation d'un rapport raffiné entre l'humain et le matériel qui produit une attraction spectaculaire. De ce fait, il n'est plus question de parler d'une rupture d'usage ou d'une complémentarité éphémère mais d'une sorte de création artistique qui se fait à l'intérieur même d'une composante quotidienne déjà structurée.

Il en résulte donc à partir de ces différentes expériences que l'objet récupéré du réel est, en grande partie, pris en considération théâtrale pour son pouvoir d'évocation d'un passé révolu avec ses différents usages, pour son déclenchement d'un imaginaire particulier unifiant la visée du spectateur avec celle du metteur en scène et pour sa mise en valeur d'une esthétique de l'usé, du trivial, voire du quotidien. D'une visée globale, il s'agit d'une réalisation théâtrale qui se base sur une poétique de l'hétérogénéité en faveur d'une réconciliation technique englobant le bricoleur, le collectionneur et le metteur en scène dans le même champ de création. Ce constat nous permet de dire que l'objet récupéré se veut le résultat d'un processus de réflexion qui met en lumière la richesse des potentialités artistiques et des performances spectaculaires des objets les plus communs. Cette nouvelle inspiration consiste à redonner une dynamique particulière à notre relation avec les éléments constituant notre univers matériel.

\section{L'esthétique de la représentation de l'objet récupéré}

L'effet produit par la représentation théâtrale de l'objet récupéré et sa nouvelle signification acquise sur la scène, nous invite à nous arrêter sur 
quelques procédés techniques qui ont favorisé une telle démarche esthétique. Il est question de relever l'apport de l'usage de l'objet récupéré sur les normes de la représentation théâtrale au niveau du décor, de l'acteur et du metteur en scène. Une telle approche révèle que ce choix esthétique de l'objet récupéré n'est pas fortuit mais il se base sur des postulats théoriques qui, pour simples qu'ils soient, redonnent à la représentation une dynamique particulière. C'est ainsi qu'on parle de l'acteur à l'image d'un conteur, du décor vraisemblable qui renforce l'authenticité de l'action dramatique et de la mise en scène cette fois-ci de la vie quotidienne.

C'est donc bien à une modification technique que les objets familiers et réels se trouvent sur la scène. Elle renforce l'idée d'un décor exact dans lequel l'acteur peut êtreen mesure de réaliser des performances au niveau de son jeu se rapportant aux gestes quotidiens dont n'importe quel spectateur maîtrise les significations. Il s'agit d'une volonté d'authentifier le jeu de l'acteur. Celui-ci peut évoluer dans un cadre vraisemblable, ce qui contribue en même temps au tissage des liens profonds non seulement entre le théâtre en tant que lieu de vision et la vie quotidienne, mais aussi, cette fois-ci notamment, en tant que source des éléments de la représentation scénique.

On pourrait éventuellement noter que cette pluralité d'usage de l'objet récupéréa dynamisé la question de la motivation de la pensée à tel point qu'on peutl'associer avec un objet de pensée par excellence. Ce constat a amené également Tadeusz Kantor (1990) à préciser que «le problème de l'art est celui de l'objet $»$. Cela nous permet de dire que toute démarche parfois révolutionnaire se rapporte à une considération particulière de l'usage de l'objet. De plus, l'esthétique de la récupération est une facette parmi d'autres qui illustrent cette orientation. Une telle esthétique nous renvoie à une remise en question relative à plusieurs considérations importantes, en l'occurrence la mémoire, la consommation et la technique.

A cet égard, Tadeusz Kantor affirme que l'objet sauvé de l'oubli nous met toujours dans une dialectique intéressante entre le passé et le présent. L'objet qui a un vécu endosse en lui des référents tangibles, ce qui redonne à la représentation une dimension réelle en facilitant la présence des caractéristiques d'une époque lointaine. Cela serait normalement difficile à réaliser avec d'autres éléments, c'est pourquoi Tadeusz Kantor parle d'une relation mystérieuse qui s'établit entre l'objet et l'historicité (Jean-luc Mattéoli, 2011: 54); "grâce au truchement des objets sauvés de l'engloutissement, le proche passé ne cesse de sourdre dans le présent, en une dialectique tendue et mystérieuse ». De ce fait, l'objet assure davantage le passage temporel et enrichit ses potentialités de jeu par un trait mémoriel en l'occurrence indéniable.

Ces objets porteurs d'une charge historique révèlent également les différentes attributions qui leur sont accordées par les utilisateurs. En effet, 
leur itinéraire d'usage reflète l'esprit de consommation qui caractérise chaque période. Dans cette perspective, Tadeusz Kantor préfère ceux qui appartiennent au rang le plus bas puisqu'ils seraient les témoins d'une réalité banale. Une telle réalité met en branle autant de processus concernant la société de consommation. Cette dernière, marquée par le changement et le renouvellement permanent de ses produits sous l'effet de la modernité, accélère en parallèle les procédés de la désuétude et de l'oubli.

Cependant, pour Tadeusz Kantor les objets récupérés même de la poubelle, déclenchent certainement une mémoire collective, ce qui remet en question nos pratiques sociales à partir d'une provocation de notre comportement matérialiste, pris dans un mouvement d'usure très accéléré. En conséquence, on parle de principes de « correction » par rapport à cette attitude distinguée par la méfiance et l'indifférence. L'intérêt est davantage porté sur la manière dont l'objet nous informe de ses différentes structures de manipulations ordinaires, esthétiques et poétiques. En outre, l'objet semble constituer la forme sur laquelle se sont opérés progressivement des modes de vie et de pensées caractérisant le système de consommation technologique et industriel.

Dès lors, il paraît que ce penchement vers l'objet usé dans les représentations théâtrales est une réaction humaine contre le changement excessif des valeurs et des normes déterminant notre civilisation urbaine. A cet égard, Tadeusz Kantor postule que «quand les systèmes de valeurs s'effondrent devant la brutalité ou la résistance du réel, les objets rebutés semblent alors être introduits plus facilement dans le champ des pratiques artistiques » (Jean-luc Mattéoli, 2007).

En se référant à cette optique, il s'agit d'un passage de l'expressivité à la chose en soi, ce qui provoque un changement cette fois-ci technique visant à considérer l'objet un élément indispensable dans la création théâtrale en dépit de sa surface de pratique quotidienne. Cette tendance s'explique de la part de Tadeusz Kantor (1990) par le fait que «nous n'avons assez de force que pour attraper ce qui était sous la main, l'objet réel, et le proclamer ouvre d'art ».

Il s'agit d'une façon de se contenter d'utiliser les éléments matériels les plus communs et surtout les proches du vécu quotidien et les considérer une création artistique. C'est un processus de représentation basé sur la simplicité et en même temps sur le rapprochement avec les principes de la vie quotidienne. Ainsi, le champ de la vie quotidienne devient une ressource à toute vision créatrice d'un imaginaire unique, une manière qui tend à lier l'objet réel et l'image artistique sous la même conception représentationnelle.

Il en ressort donc que le choix d'une telle représentation de l'objet récupéré sur la scène théâtrale est commandé par des exigences esthétiques à savoir l'historicité et l'esprit matérialiste. Dans de telles considérations, la proximité au champ du quotidien et surtout du réelsert à une construction rhétorique par excellence. En effet, la charge historique pour laquelle on identifie les 
différents usages des objets montre clairement que la touche humaine est une donnée ineffaçable et quel'évolution du temps ne peut pas complètement l'escamoter.

De la même manière l'exploitation de ce trait dans des perspectives théâtrales devient-elle un choix esthétique parce qu'elle présuppose déjà un terrain d'entente entre le spectateur et le metteur en scène à propos des visées et des thèmes à aborder. C'est de cette façon que la mémoire collective permet justement d'avancer la culture matérielle comme critère identitaire de nos comportements sociaux. Autrement-dit, le sens que l'objet récupéré véhicule dans cette situation est celui qui est partagé entre les membres de toute la société de consommation.

En effet, dans ce cas, le processus de la représentation consiste à se servir des objets, sans pour autant modifier leurs référents communs, facilement identifiables par le spectateur. En quelque sorte, la présence de l'objet induit des manières différentes au niveau de sa manipulation, mais souvent dans une configuration de gestes qui s'adaptent avec son usage familier. Sa présence permet de faire suggérer au niveau de l'imaginaire du spectateur ce que le metteur en scène vise à transmettre à partir d'un postulat qui privilégie l'ancrage à la réalité afin que la signification de l'objet ne s'efface pas de l'esprit. Cette reconfiguration se contente d'agir à partir des signes matériels.

De ce fait, le recours aux objets réelsparticipeà l'amélioration des conditions de la représentation. En ce sens, André Breton (1890) précise que ce genre d'objets;

«contribuent à la disparition de la déclamation» et remplacent " par de la composition les effets tirés uniquement de la voix [...] ; un crayon retourné, une tasse renversée, seront aussi significatifs, d'un effet aussi intense sur l'esprit du spectateur que les exagérations grandiloquentes $\gg$.

C'est l'orientation vers une expressivité concrète qui économise les longues descriptions et éclaire le jeu par des référents communs. Cela permet de dire qu'il s'agit d'une nouvelle poétique de la vie quotidienne à partir de cette reproduction minutieuse de la réalité dans l'espace scénique (Jean-luc Mattéoli, 2011: 48) «dans un monde qui va vite, le plateau offre ainsi un moyen de prendre une distance avec les images qu'il offre (par le ralentissement et l'hétérogénéité de la perception) ».

De surcroît, une chose importante à mentionnerest que le recours à l'usage de l'objet récupéré sur la scène met l'accent également sur l'habilité et la maîtrise technique de l'acteur. Ce dernier ne manipule pas un objet-outil mais un objet-témoin, ce qui lui permet de manifester une certaine adresse particulière afin de ne pas brouiller sa charge historique. En quelque sorte, l'agissement scénique de l'acteur est contrôlé par une présenceréelle et par une référence quotidienne. En ce sens, Jean Luc Mattéoli précise que l'acteur, 
par l'usage de l'objet récupéré, opère un glissement vers le statut d'un conteur. En ce sens, Jean-luc Mattéoli (2011) précise que;

"l'objet par exemple permet de raconter une multitude d'histoires; (il suffit pour cela d'accrocher son imagination à quelqu'une de ses éraflures), qui transforme le comédien en ce conteur qui avait presque disparu de nos sociétés ».

Il s'agit d'une mise en relation basée sur un discours représentatif qui stimule l'imaginaire du spectateur et offre à l'objet récupéré sa valeur théâtrale.

Ajoutons à cela un autre procédé technique dont l'importance est à souligner, celui du passage de la toile peinte au décor réel. Cette transition capitale a donné au jeu de l'acteur des possibilités diverses et au spectateur une familiarité avec l'espace de la représentation. A cet égard, André Antoine (1890) avance que "l'introduction de meubles et d'objets "vrais » en lieu et place de la toile peinte va de pair avec l'apparition de ce nouvel acteur qui « vit dans » au lieu de " jouer devant ». Un tel changement de cadre et de perspective de la vision facilite, d'une part, la mobilité et le déplacement de l'acteur sur l'espace de la scène qui se retrouve en trois dimensions et, d'autre part, l'interprétation de l'action par le spectateur qui devient une opération habituelle.

Ces différentes modifications se cristallisent clairement dans le travail du metteur en scène qui devrait agencer ces nouvelles directives dont l'objectif principal est de meubler l'espace de la représentation à l'image de la réalité. En ce sens, André Antoine (1890) postule que le metteur en scène;

«doit s'y promener longuement, y évoquer toute la vie dont il va devenir le théâtre. Il lui faudra le meubler avec sa sagacité et logique, l'orner de tous les objets familiers dont se servent, même en dehors de l'action projetée, dans les entr'actes, les habitants du lieu ».

Il s'agit, d'une certaine façon, d'opter pour une mise en scène de la réalité quotidienne dans laquelle il est question d'établir une organisation matérielle, signe d'une interaction conventionnelle entre l'acteur et le spectateur. On y trouvera presque une adéquation entre les traits distinctifs d'une structure de vie ordinaire avec celle représentée théâtralement. D'une façon générale, les choses sont telles qu'elles ont l'apparence comme celle des pratiques quotidiennes.

\section{Conclusion}

En somme, l'intérêt de la représentation par l'usage d'un objet récupéré est de porter un éclairage important sur des opérations quotidiennes qui deviennent, néanmoins, libératrices des potentialités artistiques. De plus, ces opérations sont toujours susceptibles de réaliser d'autres transformations étant 
donné quele principe catalyseur est celui du déplacement.À ce sujet, Jean-LucMattéoli (2011) affirme;

« Déplacer l'objet trouvé de la rue sur la scène, déplacer sa fonction utilitaire sur l'axe du jeu, déplacer le point de vue que le spectateur peut avoir sur le rebut, le déchet, le rien, déplacer quelques certitudes (ceci est objet), et même (comme cela s'est fait assez souvent, certes, par le passé) le lieu de la représentation, voire les spectateurs eux-mêmes ».

En toute évidence, l'objet récupéré et déplacé sur la scène donne à voir, certes, des conceptions abandonnées concernant le mouvement du temps, de 1'histoire et de la mémoire, mais il reflète simultanément un esprit de convergence entre le champ de la pratique quotidienne et celui de la représentation théâtrale.

De cette remarque, il découle que l'objectif principal de la représentation consiste à reproduire une réalité quotidienne dans un espace scénique. Restituer l'apparence d'une réalité, même ordinaire, se passe par différents moyens techniques qui vont du langage à la figuration en vue de s'approcher le plus possible de ses aspects les plus dominants. En conséquence, on peut dire que l'objet récupéré renforce l'image de notre extériorité par des référents tangibles, porteurs d'une charge historique dans un champ de la création où l'imaginaire nous fait toujours connaître d'autres formes de notre existence.

Cela nous permet de synthétiser que l'usage de l'objet récupéré sur la scène théâtrale est une considération particulière d'un élément distingué dans nos pratiques quotidiennes par sa familiarité, mais il constitue une base de départ pour une représentation théâtrale à la fois vraie et historique. Une telle réalisation audacieuse consiste à charger le rebut et le trivial par des potentialités ludiques, par des fonctionnements rhétoriques, en un mot, par une nouvelle mise en ordre du quotidien et de l'esthétique dans une réalisation théâtrale.

En quelque sorte, une nouvelle conception matérielle, prenant part à l'essentiel dans la construction du sens et dans le déroulement de l'action, va infléchir la représentation vers l'acceptation des procédés techniques aléatoires, d'assemblage et de juxtaposition. en somme, une représentation théâtrale dont l'objectif principal est de changer l'inertie relative aux éléments matériels par une dynamique dans une structure vivante qui privilégie les déplacements significatifs, l'illusion matérielle et le faire- réflexif. De ce fait, la représentation de l'objet récupéré est une façon de transformer l'inertie au vivant et de changer l'indifférence humaine contre une reconsidération artistique.

D’une visée globale, l'objet récupéré est une illustration parfaite de la liberté artistique de l'objet et de sa singularité, cette fois-ci, théâtrale. Celle-ci est acquise non seulement par une nouvelle reconnaissance de ses qualités matérielles qui ne sont pas encore exploitées, mais aussi, par un changement 
de regard envers les objets quotidiens qui peuvent servir à une représentation esthétique en catalysant le faire-perceptif du spectateur. Somme toute, l'objet récupéré articule deux instances qu'il n'est pas aisé de séparer, l'une concernant la vision qui impose un langage matériel uniquement par la présence réelle, et l'autre concernant l'imagination qui produit une telle corrélation entre des techniques systématiques et d'autres aléatoires.

Il paraît évident que ce transfert du quotidien à la représentation scénique est le reflet d'une démarche esthétique particulièrement subtile où la vision rhétorique l'emporte sur toutes considérations conventionnelles. Pour cette raison on peut résumer que le champ ludique de l'objet se trouve complètement lié aux directives ciblées au moment de la représentation et ses structures signifiantes reposent sur une manipulation rhétorique. C'est l'incarnation d'un possible théâtral, ce qui nous amène à se demander ce que pourrait être à chaque fois la structure d'apparence de l'objet et ses référents situationnels.

\section{References:}

1. Anne Ubersfeld (1984). «L'objet théâtral contemporain », Actualités des arts plastiques, CNDP, $\mathrm{N}^{\circ} 40$, « l'objet théâtral », Paris, CNDP.

2. André Antoine (1890). « Le théâtre libre », cité par Jean-Pierre Sarrazac et Philippe Marcerou, Antoine, l'invention de la mise en scène, anthologie des textes d'André Antoine (1999), Paris, Arles, Centre national du théâtre/ Actes-Sud Papiers.

3. Béatrice Picon- Vallin (1980). L'adhésion au GOSTIM, Ecrits,III , Lausanne : L'Age d'Homme, Paris, Collection.

4. Bernard Dort (1955). Théâtre populaire, Paris, Edition, seuil.

5. Bertolt Brecht (1972). Sur l'architecture scénique et la musique, Ecrits sur le théâtre, I, Paris, édition, L'Arche.

6. Bertolt Brecht (1978). Petit organon pour le théâtre, traduit par Jean Tailleur, Paris édition, L'Arche.

7. Christian Carrignon (2011). « Théâtre d'objet : mode d'emploi », Revue des arts de la scène, Agon , $\mathrm{N}^{\circ} 4$, « L'objet »:dossier artistique, mis à jour le : 25/01/2012, URL : http://agon.enslyon.fr/agon/index.php?id=2079.

8. Jean-Luc-Mattéoli (2007). « L’objet pauvre dans le théâtre contemporain », Images Re-vues, Histoire, anthropologie et théorie de l'art, $\quad \mathrm{N}^{\circ} \quad 4, \quad \ll \quad$ l'objet $», \quad$ URL : http://journals.openedition.org/imagesrevues/125.

9. Jean-Luc Mattéoli (2011). « L'objet pauvre : Mémoire et quotidien sur les scènes contemporaines françaises », Paris, Edition Presses Universitaire de Rennes. 
10. Tadeusz Kantor (1990). Leçon de Milan, traduit du polonais par MarieThérèse Vido-Rzewuska, Paris, Arles, édition, Actes sud. 